\title{
Analyzing Cement Microstructure using Multivariate Statistics
}

\author{
John Henry J. Scott and Jeffrey M. Davis \\ National Institute of Standards and Technology, Gaithersburg, MD 20899
}

Clinker and cement are chemically heterogeneous on microscopic length scales, and characterization of the spatial distribution of their distinct chemical phases is an important component of many studies in cement and concrete research. Although there are several suitable methods for characterizing coarse phases such as alite and belite, scanning electron microscopy coupled with energy-dispersive $x$-ray spectrometry (SEM-EDS) is well suited to the task of imaging and speciation of the finer-grained phases. Without direct information about the crystallographic phase present at each pixel (such as electron backscatter diffraction or x-ray diffraction data), measuring the phase distribution in cement requires a pixel classification or segmentation step where phases are assigned based on the x-ray spectral information. While conventional multichannel elemental maps have been used for this in the past, the advent of fully hyperspectral imaging systems with thousands of channels of $x$-ray data at each pixel now permits more sophisticated multivariate statistical methods to be applied to the analysis of the microstructure.

Figure 1 shows an SEM image and corresponding x-ray maps of a cross section of a mortar bar containing a highly ASR reactive aggregate, prepared according to ASTM C1260 [1]. The hardened sample was submerged in a $1 \mathrm{~N}$ solution of $\mathrm{NaOH}$. Expansion at 14 days indicated the existence of ASR reactive product, in addition to other damage to the cement microstructure [2].

Using multispectral x-ray maps based on integrated intensities of characteristic $x$-ray peaks, rulebased classification and segmentation of the image can be performed (Figure 2a), but often suffers from misclassification errors and other artifacts. Figure $2 b$ shows the result of processing the hyperspectral version of the $\mathrm{x}$-ray dataset using multivariate curve resolution via alternating least squares (MCR-ALS). This technique relies on a singular value decomposition of the entire complement of x-ray channel data to identify spectral components, significantly improving subsequent classification or segmentation steps and performing a powerful denoising function at the same time. In this sample, the spatial distribution of sodium was measured at both cryogenic and room temperature to better understand the effects of deicers on hydrated cement paste. Figure 2c is a conventional $\mathrm{x}$-ray map of the $\mathrm{Na} \mathrm{Ka}$ characteristic $\mathrm{x}$-ray fluorescence peak at $1.04 \mathrm{keV}$. Figure $2 \mathrm{~d}$ is the equivalent spectral component (also representing the spatial distribution of sodium in the field of view) resulting from the multivariate curve resolution decomposition of the dataset. Because the MCR is using the full spectral bandwidth and hyperspectral signature of the Na-rich phase, including any other elemental signatures that may be co-located with the sodium, the resulting distribution map shows much better dynamic range and less noise. This result can be used in later pixel classification and segmentation steps and translates into reduced misclassification errors and improved class separability.

\section{References}

[1] ASTM C1260, 2007 "Standard Test Method for Potential Alkali Reactivity of Aggregates (Mortar-Bar Method)", ASTM International, West Conshohocken, PA, 2007, DOI: 10.1520/C1260-07.

[2] The authors gratefully acknowledge Bryan Sudbrink of Oklahoma State University for the sample. 
[3] P. Kotula, M. Keenan, J. Michael, Microsc. Microanal. 9 (2003) 1. DOI: 10.1017/S1431927603030058

[4] (C2003 Sandia Corp.; cf. US Government Use Contract DE-AC04-4AL85000, License \# 04-N01278.2.
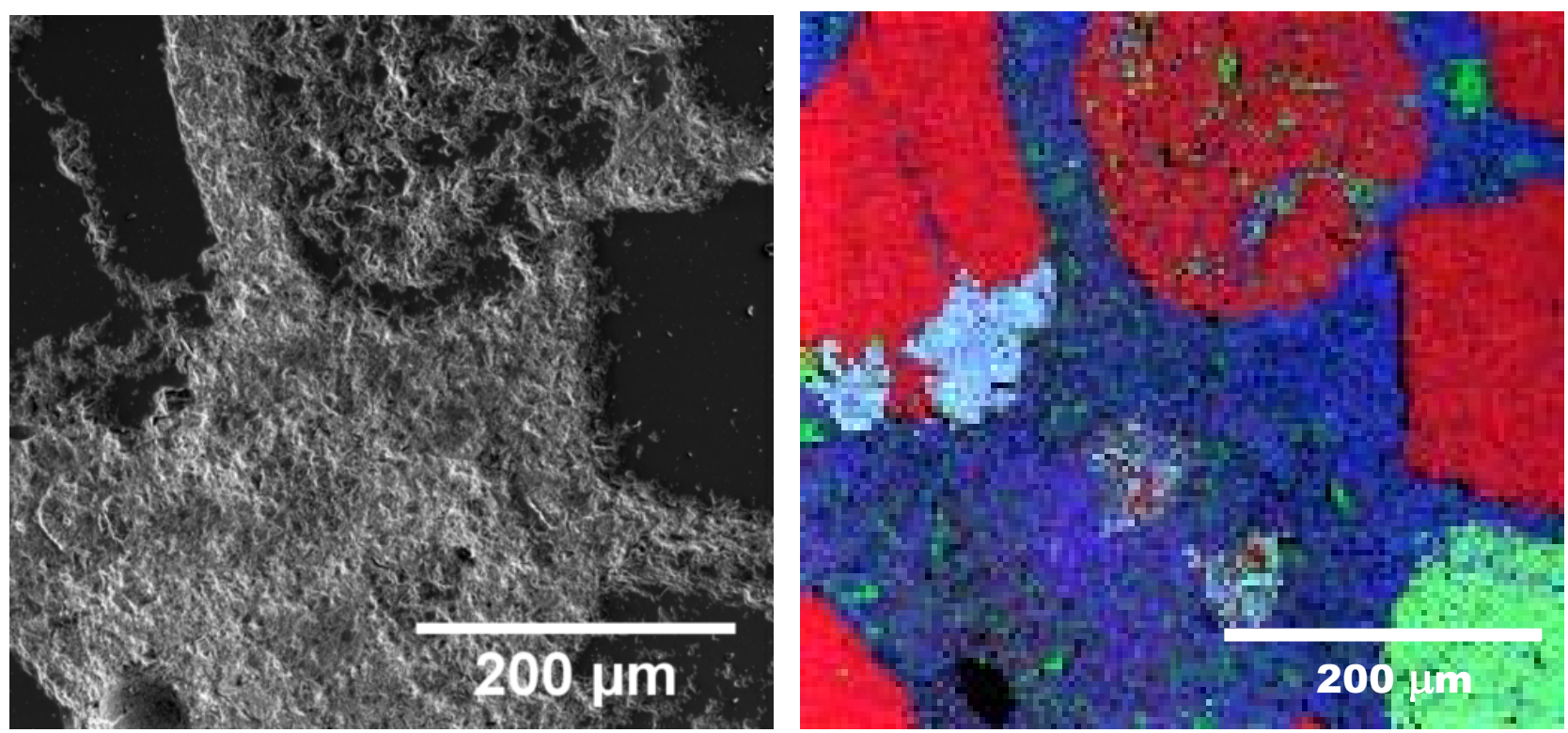

Figure 1. Scanning electron micrograph of cement displaying structural heterogeneity at several length scales (left), and an energy dispersive x-ray map of the same field of view (right). The electron micrograph was produced using the Everhart-Thornley detector, while in the x-ray map the false colors red, green, and blue represent fluoresced x-ray intensities in $130 \mathrm{eV}$ wide windows centered on $1.74 \mathrm{keV}(\mathrm{Si} \mathrm{Ka}), 1.49 \mathrm{keV}(\mathrm{Al} \mathrm{Ka})$, and $1.04 \mathrm{keV}(\mathrm{Na} \mathrm{Ka})$ respectively. Horizontal full width of each image is 478 micrometers.
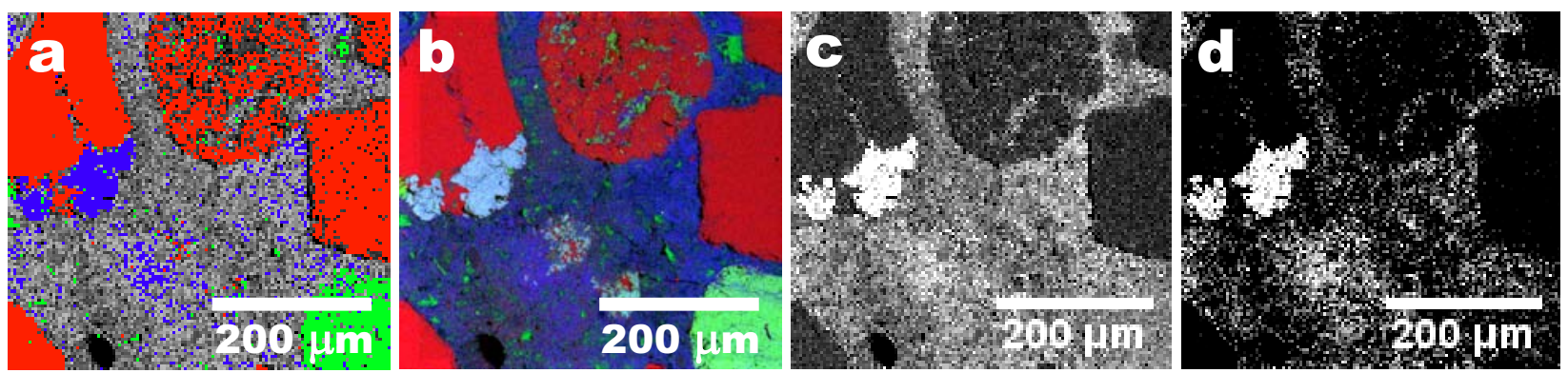

Figure 2. (a) Pixel classification using individual element $\mathrm{x}$-ray map thresholds (red=Si, green=Al, blue $=\mathrm{Na}$ ); (b) false color map showing the intensities of three spectral components derived using multivariate curve resolution (MCR); (c) single element x-ray intensity map for $\mathrm{Na} \mathrm{Ka}$; (d) intensity map of the equivalent spectral component derived using singular value decomposition (SVD) followed by a spatial simplicity rotation in AXSIA [3,4]; note the much higher signal-to-noise ratio, which translates directly into fewer pixel misclassification errors. 